\title{
Relationship of common variants in Krüppel-like factor 7 gene with susceptibility and prognosis of oral squamous cell carcinoma
}

\section{Lili Wang}

Chinese PLA General Hospital

Hongguang Song

Beijing DCN Orthopaedic Hospital

Shiming Yang ( $\square$ sujoain@yeah.net)

Chinese PLA General Hospital https://orcid.org/0000-0002-8413-5054

Research article

Keywords: Krüppel-like factor 7, Oral squamous cell carcinoma, Prognosis

Posted Date: September 8th, 2020

DOI: https://doi.org/10.21203/rs.3.rs-61631/v1

License: (c) (i) This work is licensed under a Creative Commons Attribution 4.0 International License.

Read Full License 


\section{Abstract \\ Background}

The aim of this study was to assess the prognostic value of Krüppel-like factor 7 (KLF 7 ) for patients with oral squamous cell carcinoma(OSCC).

\section{Methods}

The expression of KLF7 was detected by quantitative real-time polymerase chain reaction (qRT-PCR) in pairs of tumor tissues and adjacent non-tumor tissues of OSCC. Chi-square $\left(\chi^{2}\right)$ test was applied to evaluate the association of KLF7 expression with clinicopathological characteristics of OSCC patients. Overall survival was estimated using the Kaplan-Meier method with log rank test. The cox proportional hazards model was used for univariate and multivariate analyses.

\section{Results}

The expression of $K L F 7$ was remarkably increased in OSCC tissues compared with adjacent non-tumor tissues $(P<0.001)$. KLF7 expression was related to TNM stage $(P=0.006)$, tumor size $(P=0.010)$, smoking $(P=0.006)$ and drinking $(P=0.000)$. Kaplan-Meier analysis showed that OSCC patients with high KLF7 expression had a poorer overall survival than those with low expression (log rank test, $P=$ 0.018). Moreover, multivariate analyses showed that $K L F 7$ was an independent prognostic factor for $\operatorname{OSCC}(P=0.002 \mathrm{HR}=2.64595 \% \mathrm{Cl}: 1.426-4.906)$.

\section{Conclusion}

Decreased expression of $K L F 7$ may be a potential unfavorable prognostic factor for patients with OSCC.

\section{Background}

Oral squamous cell carcinoma (OSCC) is one of the most common forms of oral cancer worldwide, and its morbidity has been rapidly increasing in Asian countries in the past decade [1]. There are several risk factors confirmed for patients with OSCC, such as tobacco smoking, alcohol consumption, human papillomavirus infection, etc [2-4]. The conventional treatments for OSCC include surgical treatment, chemotherapy, radiotherapy. In the past few years, the therapeutic strategies for OSCC have been significantly improved, but the five-year survival of the patients still remains unsatisfactory, especially those diagnosed with advanced stages [5-7]. The aggressive tumor progression and high recurrence rate may be responsible for the poor clinical outcomes [8]. Until now, OSCC progression evaluation is mainly dependent on TNM staging. However, the TNM system is based on clinical parameters of the cases. The histopathological features as well as the interaction between the tumor and host are not considered, 
which may cause bias to the final results [9]. Therefore, novel biomarkers are in urgent need to evaluate tumor progression and guide treatments in patients with OSCC.

Krüppel-like factors (KLFs) are a group of DNA-binding transcriptional regulators which contain zinc fingers. The family plays important roles in a variety of biological processes, such as cell stemness, proliferation, differentiation, apoptosis, and energy metabolism [10-12]. Krüppel-like factor 7 (KLF7) is a member of the KLF family, and three $\mathrm{C} 2 \mathrm{H} 2$ type zinc fingers are located at its $\mathrm{C}$-terminus that bind to the promoters of target genes [13]. KLF7 could regulate the function of the nervous system and adipose tissue [14]. Knockdown the expression of KLF7 may cause damages to neuronal and cardiomyocytic differentiation of embryonic stem cells [15]. Besides, the abnormal expression of KLF7 may be also involved in regulation of blood disease, type 2 diabetes, obesity, etc [16-18]. Recently, tumor investigations have demonstrated that abnormal expression of $K L F 7$ might be involved in carcinogenesis. For example, Ding et al. reported that the expression of $K L F 7$ was up-regulated in OSCC tissues, moreover, its elevated expression showed positive association with the migration ability of OSCC cells. KLF7 might serve as a promoter in metastasis of OSCC [19]. However, to our knowledge the prognostic significance of $K L F 7$ in OSCC remained unclear.

In the present study, we analyzed the relative expression level of KLF7 in OSCC carcinoma tissues, as well as its association with clinicopathological factors. In addition, a five-year follow up investigation was performed for the patients, and the clinical significance of $K L F 7$ for prognosis prediction in OSCC patients was also investigated in the current study.

\section{Materials And Methods}

\section{Patients and tissue samples}

The study was approved by the Ethic Committee of Chinese PLA General Hospital. All patients provided written informed consents in advance.

In the present study, a total of 111 OSCC cases were recruited from Chinese PLA General Hospital. All patients were pathologically diagnosed with OSCC and did not receive any treatment prior. The tumor tissues and adjacent non-cancerous tissue specimens were collected from each patient, then the specimens were immediately frozen in liquid nitrogen and stored at $-80^{\circ} \mathrm{C}$ until use.

All the patients were enrolled in a five-year follow up investigations. The survival information during the follow-up was recorded for survival analysis. The patients whose death was not related to OSCC would be excluded from the study.

\section{RNA extraction and quantitative real-time polymerase chain reaction (qRT-PCR)}


Total RNA was extracted from OSCC tumor specimens and matched adjacent non-tumor tissues using TRIzol reagent (Invitrogen) according to the manufacturer's procedure. The expression level of KLF7 was determined by qRT-PCR, and the reaction was performed with the SYBR® Green dye (TaKaRa, Dalian, China) on the 7500 Real-Time PCR systems (Applied Biosystems, Carlsbad, CA, USA). $\beta$-actin served as internal control. The primer sequences were as follows: KLF7 forward (F), 5'-ACTGCTTGCTGACAATCTCG$3^{\prime}$ and reverse (R), 5'-GGTCCCTCACACATCCTTCA-3'; $\beta$-actin F, 5'-TGACGTGGACATCCGCAAAG-3' and R, 5'CTGGAAGGTGGACAGCGAGG-3'. The fold-change for target genes normalized by internal control was determined by $2^{-\triangle \triangle C T}$ method. Each sample was examined in triplicate.

\section{Statistical analysis}

Statistical analysis were performed using SPSS statistics 18.0 (SPSS, Chicago, IL, USA) and the graph was plotted using GraphPad Prism 5.0 (GraphPad Software Inc., USA). The expression value of KLF7 in tissue specimens were shown as mean \pm standard deviation (SD), and its comparison between tumor and non-cancerous specimens were carried out using student's $t$ test. The relationship between KLF7 expression levels and clinicopathologic characteristics was estimated by chi-square test. Kaplan-Meier curve was applied to analyze the effects of $K L F 7$ expression on overall survival of OSCC cases. The prognostic value of $K L F 7$ in OSCC was evaluated using Cox regression model. The $P$ values less than 0.05 were considered statistically significant.

\section{Results}

\section{The expression level of KLF7 in OSCC}

A total of 111 OSCC cases including 53 females and 58 males were collected in our study. QRT-PCR were used to investigate the expression pattern of $K L F 7$ in the OSCC tissues and adjacent non-tumor tissues. The results showed that the relative expression of $K L F 7$ was significantly increased in OSCC tissues compared with adjacent non-tumor tissues $(P<0.001)$ (Fig. 1).

\section{The relationships between KLF7 expression and clinicopathological features}

To assess the correlation of $K L F 7$ expression with clinicopathological parameters of patients, the patients were divided into high $K L F 7$ expression group $(n=56)$ and low $K L F 7$ expression group $(n=55)$ based on their mean expression value. The expression levels of $K L F 7$ were not correlated with age and gender (all $P>0.05$ for both) (Table 1). However, the patients with high expression of $K L F 7$ were more likely to exhibit advanced TNM stage $(P=0.006)$, large tumor size $(P=0.010)$, smoking $(P=0.006)$ and drinking $(P=$ 0.000) (Table 1). 
Table 1

The correlation between KLF7 expression and clinicopathological features of OSCC patients

\begin{tabular}{|c|c|c|c|c|}
\hline \multirow[t]{2}{*}{ Features } & \multirow{2}{*}{$\begin{array}{l}\text { NO. of cases } \\
(n=111)\end{array}$} & \multicolumn{2}{|c|}{ KLF7 expression } & \multirow[t]{2}{*}{$P$ values } \\
\hline & & Low $(n=55)$ & High $(n=56)$ & \\
\hline Age (years) & & & & 0.218 \\
\hline$<60$ & 48 & 27 & 21 & \\
\hline$\geq 60$ & 63 & 28 & 35 & \\
\hline Gender & & & & 0.298 \\
\hline Female & 53 & 29 & 24 & \\
\hline Male & 58 & 26 & 32 & \\
\hline Smoking status & & & & 0.006 \\
\hline None-smoker & 51 & 18 & 33 & \\
\hline Smoker & 60 & 37 & 23 & \\
\hline Tumor size $(\mathrm{cm})$ & & & & 0.010 \\
\hline$<5$ & 52 & 19 & 33 & \\
\hline$\geq 5$ & 59 & 36 & 23 & \\
\hline Drinking status & & & & 0.000 \\
\hline None-drinker & 58 & 18 & 40 & \\
\hline Drinker & 53 & 37 & 16 & \\
\hline TNM stage & & & & 0.006 \\
\hline \-】 & 53 & 19 & 34 & \\
\hline 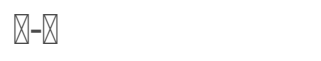 & 58 & 36 & 22 & \\
\hline
\end{tabular}

\section{The prognostic value of KLF7 expression in OSCC}

In order to investigate the prognostic value of KLF7 expression for OSCC, we assessed the association between KLF7 expression levels and patient survival using Kaplan-Meier analysis with log-rank test. Survival analysis indicated that OSCC patients with high KLF7 expression had worse overall survival than those with low $K L F 7$ expression (log rank test, $P=0.018$ ) (Fig. 2). Univariate analysis revealed that $K L F 7$ expression $(P=0.024)$, TNM stage $(P=0.029)$, smoking status $(P=0.026)$ and drinking status $(P=0.026)$ were potential prognostic indicators for OSCC patient. As shown in Table 2, multivariate analysis confirmed $K L F 7$ expression $(P=0.002 \mathrm{HR}=2.64595 \% \mathrm{Cl}$ : $1.426-4.906)$ and smoking status $(P=0.002$; $\mathrm{HR}=2.626 ; 95 \% \mathrm{Cl}: 1.415-4.871)$ were independently correlated with prognosis of OSCC patients. 
Table 2

The univariate and multivariate analyses for patients with OSCC

\begin{tabular}{|lllll|}
\hline Factors & \multicolumn{3}{l}{ Univariate analysis } & \multicolumn{3}{l|}{ Multivariate analysis } \\
\cline { 2 - 5 } & $\mathrm{HR}(95 \% \mathrm{Cl})$ & $\boldsymbol{P}$ values & $\mathrm{HR}(95 \% \mathrm{Cl})$ & $P$ values \\
\hline KLF7 expression & $1.989(1.096-3.608)$ & 0.024 & $2.645(1.426-4.906)$ & 0.002 \\
\hline Age (years) & $1.555(0.856-2.828)$ & 0.147 & - & - \\
\hline Gender & $1.278(0.719-2.272)$ & 0.404 & - & - \\
\hline Smoking status & $2.065(1.090-3.915)$ & 0.026 & - & - \\
\hline Drinking status & $1.970(1.085-3.574)$ & 0.026 & $2.626(1.415-4.871)$ & 0.002 \\
\hline Tumor size (cm) & $1.033(0.575-1.853)$ & 0.914 & - & - \\
\hline TNM stage & $2.040(1.076-3.868)$ & 0.029 & - & - \\
\hline
\end{tabular}

\section{Discussion}

Oral squamous cell carcinoma (OSCC) is an aggressive human malignancy worldwide. Despite advances in prevention and treatment, the five-year survival rate of OSCC patients remains low due to metastasis and local recurrence $[8,20]$. Tumor progression and prognosis evaluations are a great challenge for OSCC patients in clinic, due to the lack of effective and reliable biomarkers. Growing evidences have demonstrated that genetic factors play a pivotal role in progression of OSCC. Therefore, to investigate the OSCC-related genes may explore novel and effective biomarkers for patients with OSCC. In the present study, we investigated the prognostic value of KLF7 in OSCC patients.

KLFs are a family of zinc-finger transcription factors, which are widely expressed in multiple human organs or tissues [21]. KLF family is involved in diverse biological processes, and their abnormal expression may lead to human diseases, like cancer [22]. In oral cancer, several members of the KLFs family had been proved to be implicated in the pathogenesis of the cancers. For examples, Uchida $D$ et al. had demonstrated that knockdown the expression of $K L F 2$ would achieve the anti-tumor effects in oral cancer cells [23]. KLF4 and KLF5 might regulate the dedifferentiation and differentiation of oral carcinoma cells, thus leading to oral cancer carcinogenesis [24-26]. KLF8 was up-regulated in oral cancer cells, and its down-regulation might suppress the proliferation and clone formation of the cells [27]. In addition, the cellular levels of $K L F 13$ was significantly correlated with proliferation ability and therapy sensitivity of oral cancer, which might be a potential diagnostic biomarker and therapeutic target for oral cancer [28]. However, to our knowledge, the function of $K L F 7$ as well as its clinical significance had been rarely reported in OSCC.

In the present study, we found that KLF7 expression levels were up-regulated in OSCC tissues compared with adjacent non-tumor tissues. Additionally, the expression of $K L F 7$ was positively correlated with tumor size, TNM stage, smoking status and drinking status. This data indicated that KLF7 might be 
involved in the development of OSCC. High expression of KLF7 predicted malignant clinical characteristics for the patients with OSCC. The study scheduled by Ding et al. have demonstrated that over-expression of KLF7 via mediating the expression of snail enhanced the migratory potential of OSCC cells, and induced epithelial-mesenchymal transition (EMT) and lymph node metastasis [19]. However, the carcinogenic mechanisms of KLF7 in OSCC was not investigated in the current study. Further relevant researches were still needed to address the issues.

Given its functional roles in OSCC development, we investigated the clinical performance of KLF7in prognosis evaluation in OSCC population. Kaplan-Meier survival analysis with log-rank test suggested that high-expression group had obviously shorter overall survival than the low-expression group. Multivariate analysis with a Cox proportional hazards model indicated that high KLF7 expression was independently linked to poor survival of patients with OSCC. KLF7 might serve as an independent prognostic factor for patients with OSCC. Although we confirmed the prognostic value of KLF7 in OSCC, there were several limitations in our study. First, the sample size was relatively small. Second, only one single population was enrolled in our researches. Due to the diverse genetic background, the expression profile of $K L F 7$ in other races might be different. In addition, despite of the diverse biomarkers confirmed for OSCC, few of then were applied in clinic. Thus, subsequent investigations still required to analyze the clinical application of $K L F 7$ for OSCC cases.

\section{Conclusions}

In conclusion, the expression of $K L F 7$ is increased in OSCC, and shows positive association with aggressive clinical parameters of OSCC cases. KLF7 may be an independent biomarker for prognosis prediction in OSCC.

\section{Abbreviations}

Krüppel-like factor 7 (KLF入)

oral squamous cell carcinoma(OSCC)

quantitative real-time polymerase chain reaction (qRT-PCR)

Krüppel-like factors (KLFs)

standard deviation (SD)

epithelial-mesenchymal transition (EMT)

\section{Declarations}

\section{Ethics approval and consent to participate}


This study was supported by the Ethics Committee of Chinese PLA General Hospital and also has been carried out in accordance with the World Medical Association Declaration of Helsinki.

The subjects had been informed the objective. Certainly, written consents were signed by every subject in this study.

\section{Consent for publication}

We obtaining permission from participants to publish their data.

\section{Availability of data and materials}

All data generated or analysed during this study are included in this published article.

\section{Competing interests}

The authors declare that they have no competing interests.

\section{Authors' contributions}

L.W. design of the work; L.W. the acquisition, analysis, H.S. interpretation of data; H.S. the creation of new software used in the work; S.Y. have drafted the work or substantively revised it. All authors read and approved the final manuscript.

\section{Acknowledgements}

Not applicable.

\section{References}

1. Krishna Rao SV, Mejia G, Roberts-Thomson K, Logan R. Epidemiology of oral cancer in Asia in the past decade-an update (2000-2012). Asian Pacific journal of cancer prevention: APJCP. 2013;14(10):5567-77.

2. Chou YE, Hsieh MJ, Hsin CH, Chiang WL, Lai YC, Lee YH, Huang SC, Yang SF, Lin CW. CD44 gene polymorphisms and environmental factors on oral cancer susceptibility in Taiwan. PloS one. 2014;9(4):e93692.

3. Chuang CY, Chen MK, Hsieh MJ, Yeh CM, Lin CW, Yang WE, Yang SF, Chou YE. High Level of Plasma EGFL6 Is Associated with Clinicopathological Characteristics in Patients with Oral Squamous Cell Carcinoma. International journal of medical sciences. 2017;14(5):419-24.

4. Malik UU, Zarina S, Pennington SR. Oral squamous cell carcinoma: Key clinical questions, biomarker discovery, and the role of proteomics. Archives of oral biology. 2016;63:53-65.

5. Winck FV, Prado Ribeiro AC, Ramos Domingues R, Ling LY, Riano-Pachon DM, Rivera C, Brandao TB, Gouvea AF, Santos-Silva AR, Coletta RD, et al. Insights into immune responses in oral cancer through 
proteomic analysis of saliva and salivary extracellular vesicles. Scientific reports. 2015;5:16305.

6. van der Waal I. Are we able to reduce the mortality and morbidity of oral cancer, some considerations. Medicina oral, patologia oral y cirugia bucal 2013, 18(1):e33-37.

7. Fang J, Li X, Ma D, Liu X, Chen Y, Wang Y, Lui VWY, Xia J, Cheng B, Wang Z. Prognostic significance of tumor infiltrating immune cells in oral squamous cell carcinoma. BMC Cancer. 2017;17(1):375.

8. Abdul-Aziz MA, Amin AK, El-Rouby DH, Shaker OG. Lymphangiogenesis in Oral Squamous Cell Carcinoma: Correlation with VEGF-C Expression and Lymph Node Metastasis. Int J Dent. 2017;2017:7285656.

9. Cortegoso AVB, Laureano NK, Silva ADD, Danilevicz CK, Magnusson AS, Visioli F, Rados PV. Cell proliferation markers at the invasive tumor front of oral squamous cell carcinoma: comparative analysis in relation to clinicopathological parameters of patients. Journal of applied oral science: revista FOB. 2017;25(3):318-23.

10. Bieker JJ. Kruppel-like factors: three fingers in many pies. J Biol Chem. 2001;276(37):34355-8.

11. Zhang Z, Wang H, Sun Y, Li H, Wang N. Klf7 modulates the differentiation and proliferation of chicken preadipocyte. Acta Biochim Biophys Sin. 2013;45(4):280-8.

12. McConnell BB, Yang VW. Mammalian Kruppel-like factors in health and diseases. Physiological reviews. 2010;90(4):1337-81.

13. Wang X, Shen QW, Wang J, Zhang Z, Feng F, Chen T, Zhang Y, Wei H, Li Z, Wang Y. KLF7 Regulates Satellite Cell Quiescence in Response to Extracellular Signaling. Stem Cells. 2016;34(5):1310-20.

14. Chen YC, Wei H, Zhang ZW. [Research progress of Kruppel-like factor 7]. Sheng Li Xue Bao. 2016;68(6):809-15.

15. Caiazzo M, Colucci-D'Amato L, Esposito MT, Parisi S, Stifani S, Ramirez F, di Porzio U. Transcription factor KLF7 regulates differentiation of neuroectodermal and mesodermal cell lineages.

Experimental cell research. 2010;316(14):2365-76.

16. Schuettpelz LG, Gopalan PK, Giuste FO, Romine MP, van Os R, Link DC. Kruppel-like factor 7 overexpression suppresses hematopoietic stem and progenitor cell function. Blood. 2012;120(15):2981-9.

17. Kawamura Y, Tanaka Y, Kawamori R, Maeda S. Overexpression of Kruppel-like factor 7 regulates adipocytokine gene expressions in human adipocytes and inhibits glucose-induced insulin secretion in pancreatic beta-cell line. Mol Endocrinol. 2006;20(4):844-56.

18. Zobel DP, Andreasen CH, Burgdorf KS, Andersson EA, Sandbaek A, Lauritzen T, Borch-Johnsen K, Jorgensen T, Maeda S, Nakamura Y, et al. Variation in the gene encoding Kruppel-like factor 7 influences body fat: studies of 14818 Danes. Eur J Endocrinol/Eur Fed Endocr Soc. 2009;160(4):603-9.

19. Ding X, Wang X, Gong Y, Ruan H, Sun Y, Yu Y. KLF7 overexpression in human oral squamous cell carcinoma promotes migration and epithelial-mesenchymal transition. Oncology letters. 2017;13(4):2281-9. 
20. Taghavi N, Yazdi I. Prognostic factors of survival rate in oral squamous cell carcinoma: clinical, histologic, genetic and molecular concepts. Arch Iran Med. 2015;18(5):314-9.

21. Sun F, Hu K. Kruppel-Like Factor 4 Inhibits the Transforming Growth Factor-beta1-Promoted Epithelial-to-Mesenchymal Transition via Downregulating Plasminogen Activator Inhibitor-1 in Lung Epithelial Cells. Disease markers. 2015;2015:473742.

22. Wang X, Jiang Z, Zhang Y, Liu L, Fan Z. RNA sequencing analysis reveals protective role of kruppellike factor 3 in colorectal cancer. Oncotarget. 2017;8(13):21984-93.

23. Uchida D, Onoue T, Begum NM, Kuribayashi N, Tomizuka Y, Tamatani T, Nagai H, Miyamoto Y. Vesnarinone downregulates CXCR4 expression via upregulation of Kruppel-like factor 2 in oral cancer cells. Mol Cancer. 2009;8:62.

24. Li W, Liu M, Su Y, Zhou X, Liu Y, Zhang X. The Janus-faced roles of Kruppel-like factor 4 in oral squamous cell carcinoma cells. Oncotarget. 2015;6(42):44480-94.

25. Shibata M, Chiba T, Matsuoka T, Mihara N, Kawashiri S, Imai K. Kruppel-like factors 4 and 5 expression and their involvement in differentiation of oral carcinomas. Int $\mathrm{J}$ Clin Exp Pathol. 2015;8(4):3701-9.

26. Kim CK, He P, Bialkowska AB, Yang VW. SP and KLF Transcription Factors in Digestive Physiology and Diseases. Gastroenterology. 2017;152(8):1845-75.

27. Bin Z, Ke-Yi L, Wei-Feng Z, Li-Cheng J, Xian-Bin L, Chun-Peng X, Dao-Ying Y, Shu-Wei L: Downregulation of KLF8 expression by shRNA induces inhibition of cell proliferation in CAL27 human oral cancer cells. Medicina oral, patologia oral y cirugia bucal 2013, 18(4):e591-596.

28. Henson BJ, Gollin SM. Overexpression of KLF13 and FGFR3 in oral cancer cells. Cytogenet Genome Res. 2010;128(4):192-8.

\section{Figures}




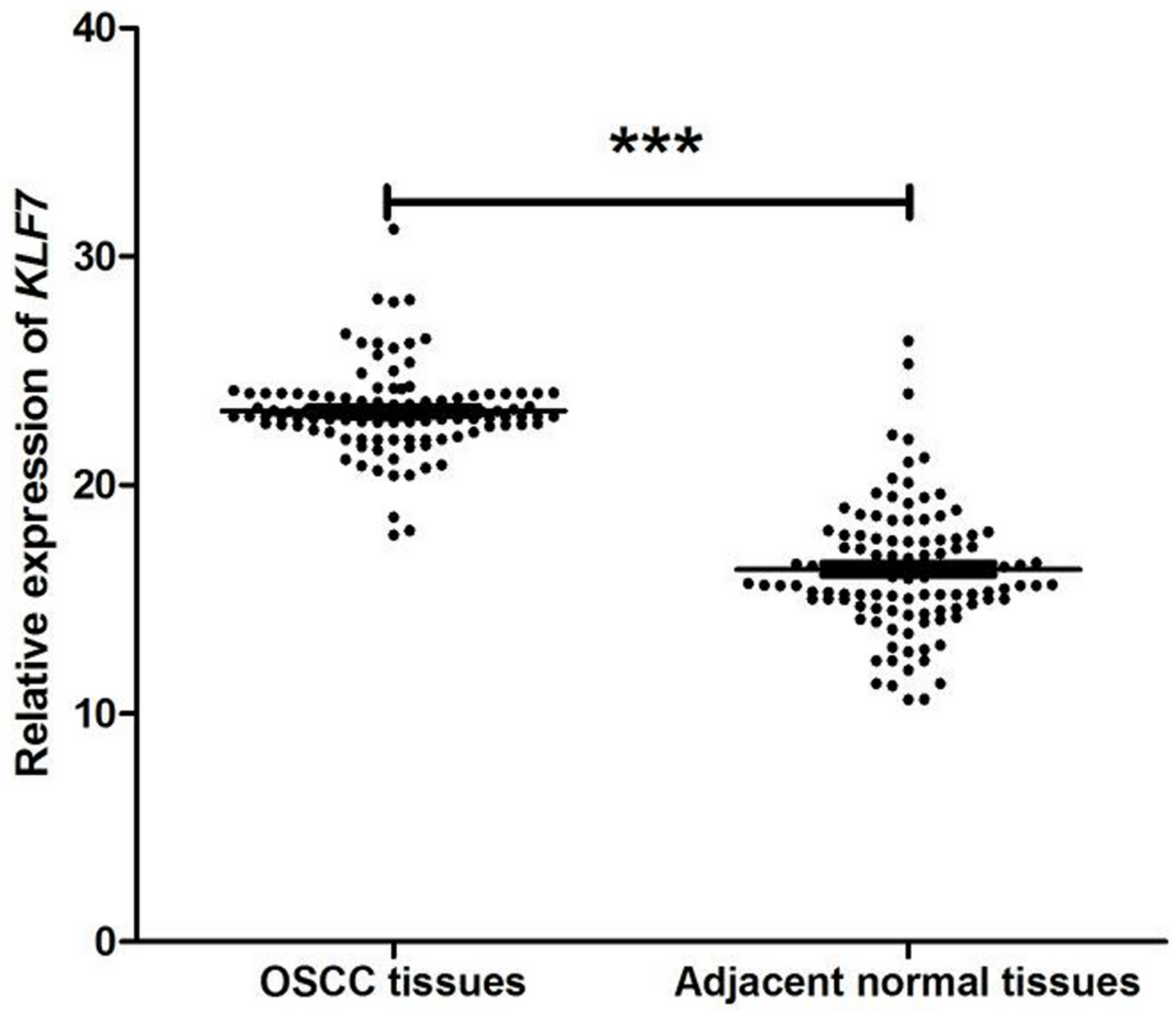

Figure 1

The relative expression of KLF7 in OSCC patients was detected using qRT-PCR. Results showed that KLF7 expression was significantly increased in OSCC tissues compared with adjacent non-tumor tissues (***: indicated $\mathrm{P}<0.001)$. 


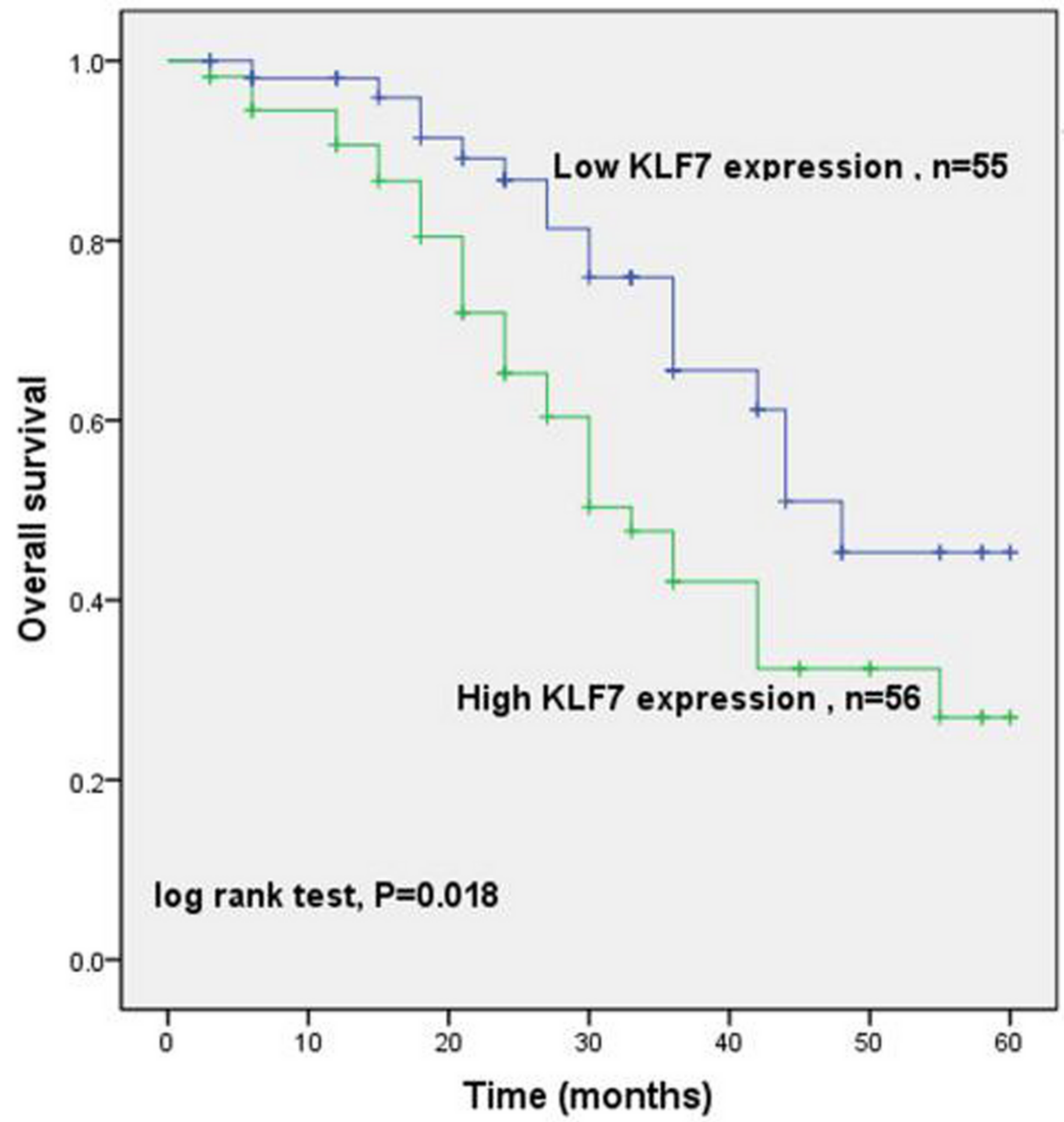

Figure 2

Kaplan-Keier survival curves of OSCC patients based on KLF7 expression levels. Patients with high KLF7 expression had significantly poorer prognosis than those with low expression (log rank test, $\mathrm{P}=0.018$ ). 